\title{
Tunable local polariton modes in semiconductors*
}

\author{
M. Foygel, ${ }^{1,2}$ Alexey Yamilov, ${ }^{1}$ Lev I. Deych,${ }^{3}$ and A. A. Lisyansky ${ }^{1}$ \\ 1 Department of Physics, Queens College of CUNY, Flushing, NY 11367 \\ 2 Department of Physics, South Dakota School Mines and Technology, Rapid City, SD 57701 \\ ${ }^{3}$ Department of Physics, Seton Hall University, South Orange, NJ 07079
}

(November 3, 2018)

\begin{abstract}
We study the local states within the polariton bandgap that arise due to deep defect centers with strong electron-phonon coupling. Electron transitions involving deep levels may result in alteration of local elastic constants. In this case, substantial reversible transformations of the impurity polariton density of states occur, which include the appearance/disappearance of the polariton impurity band, its shift and/or the modification of its shape. These changes can be induced by thermo- and photoexcitation of the localized electron states or by trapping of injected charge carriers. We develop a simple model, which is applied to the $O_{P}$ center in $G a P$. Further possible experimental realizations of the effect are discussed.
\end{abstract}

\section{INTRODUCTION}

Capture of non-equilibrium charge carriers by a deep defect center provides an important channel of energy dissipation in wide-bandgap semiconductors and insulators. 1 A significant amount of energy, at least equal to the binding energy, $\epsilon_{T} \simeq 1 \mathrm{eV}$, of the electron (or the hole) to the center, should be released in each capture event, is usually accompanied by a substantial lattice relaxation. Several mechanisms can be responsible for the electron transitions involving deep levels in semiconductors. The energy lost by the captured carrier can be transferred either to photon(s) in the radia tive transition, 2 or to nearby carrier in the Auger effect, or to a series of long-wavelength acoustic phonons when the carrier descends a staircase of the excited states in the cascade mechanism, 6 or to the local vibration quanta when multi-phonon emission 3 takes place. There are some indirect 6 and direct $2 \mathrm{t}$ evidence that capture or release of the charge carrier is associated not only with the lattice relaxation but, more importantly with the alteration of local vibrational modes (LVM's), i.e. with changes in the local elastic constants.

The subject of the present paper is local excitations of a different type, local polariton modes (LPM's), that are sensitive to the charge-state induced changes in local elastic constants. These modes arise in polar crystals in the vicinity of a polariton resonance, where a strong phononlight interaction results in splitting of the longitudinal (LO) and transverse (TO) optic modes. If the spatial dispersion of the TO modes is negative in all crystallographic directions, there exists a frequency region where the density of polaritons states vanishes. Defects introduced in such a lattice may then lead to LPM's inside the polariton bandgap.

LPM's associated with substitutional defects were introduced in Refs. 19,20. They represent electromagnetic excitations coupled to phonons or excitons with both components, including the electromagnetic component, localized in the vicinity of the defect. Although LVM's also interact with the external electromagnetic field, this interaction results mainly in resonance scattering of light and radiative decay of the states. Contrary to LVM's, LPM's arise in the polariton bandgap, where electromagnetic waves cannot propagate. Therefore, there is neither defect-induced scattering of light nor radiative damping of the local states. LPM's lead to new optical effects and strongly affect the properties of impure crystals.19 22

In this paper, we show that electron transitions involving deep centers in semiconductors may lead to a reversible changes of the frequency of the existing LPM's. We also discuss an even more interesting possibility of creating/eliminating LPM's by changing the charge-state of a deep center. When the concentration of these centers is sufficiently high LPM's develop into an impurity polariton band (IPB) 25, 26 We show that in this case the alteration of the local elastic constants can lead to the creation of an IPB or to the shift of the existing band (and/or to alteration of its shape). We review materials where these effects may be observed experimentally.

\section{LOCAL POLARITON STATES}

The system under consideration is a polar 3D crystal where dynamics of the atoms can be described by the classical Newton equations. Polaritons in the system arise as collective excitations of the polarization waves related to optical phonons of "right" symmetry, coupled to the electromagnetic field by means of a coupling parameter $\alpha$ proportional to the oscillator strength of the respective oscillations. The electromagnetic subsystem is described by Maxwell equations that include the polarization density related to phonons 20

${ }^{*}$ Submitted to PRB 
In a perfect crystal, the solution of the system of the Maxwell and atomic equations in the long-wave approximation yields the dispersion equation. The dispersion curves, of course, depend on the symmetry of the crystal. For our consideration, however, the particular form of the dispersion is not important as long as the polariton gap exists. Therefore, in the long-wave approximation we can present the upper, $\Omega_{+}(k)$, and lower, $\Omega_{-}(k)$, polariton branches in the following isotropic form 20 (see Fig. $1)$ :

$$
\begin{aligned}
\Omega_{ \pm}^{2}(k) & =\frac{1}{4}\left(\sqrt{\left[\Omega_{\perp}(k)+c k\right]^{2}+d^{2}}\right. \\
& \left. \pm \sqrt{\left[\Omega_{\perp}(k)-c k\right]^{2}+d^{2}}\right)^{2}
\end{aligned}
$$

Here $\Omega_{\perp}^{2}(k)$ is the TO branch of the phonon spectrum, which in the long-wave limit can be approximated as $\Omega_{0}^{2}-v_{\perp}^{2} k^{2}$, where $v_{\perp}$ determines the spatial dispersion of TO phonons, $c$ is the speed of light in the crystal, and $k$ is the wave vector. The width of the polariton bandgap, $\left(\Omega_{0}, \sqrt{\Omega_{0}^{2}+d^{2}}\right)$, is determined by a parameter $d$, related to the coupling constant $\alpha$.

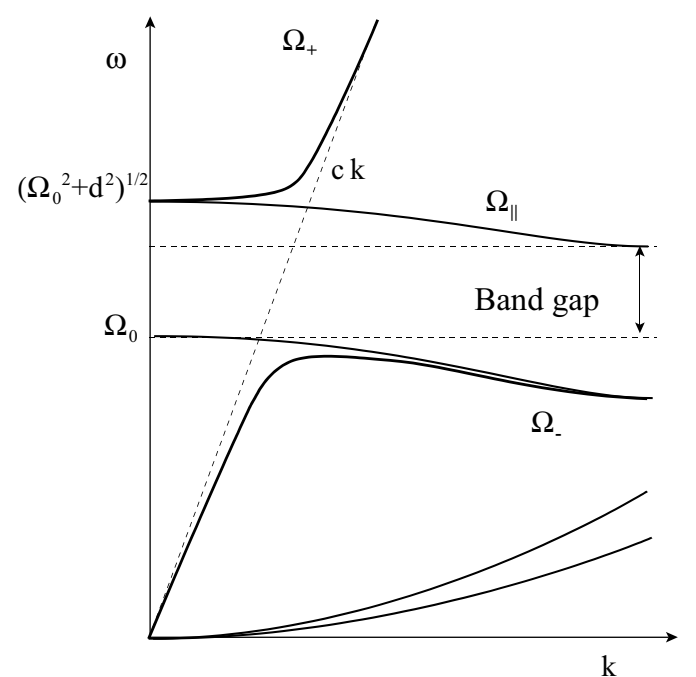

FIG. 1. Schematic phonon dispersion curves in a polar crystal.

In ideal crystals, LO phonons do not interact with transverse excitations. In the presence of defects, however, restrictions due to momentum conservation are relaxed, and the energy of LPM can leak via LO phonons, if the latter have a nonzero density of states at the frequency of LPM. In this case, the phonon component of LPM becomes delocalized, but its electromagnetic component remains localized. There are few crystals where the dispersion of the LO branch, $\Omega_{\|}^{2}(k)=$ $\sqrt{\Omega_{0}^{2}+d^{2}}-v_{\|}^{2} k^{2}$, is not large enough to fill the entire bandgap and LPM can exist as truly localized states. In most cases, the LO modes have rather large dispersion with a non-zero density of the phonon states throughout the entire gap. However, the large dispersion leads to a relatively small density of the LO states, and the lifetimes of LPM's in certain materials can be large enough for their survival.26

The equation for the frequency of LPM, $\Omega_{l o c}$, in the presence of a substitutional defect in a two-sublattice crystal was obtained in Ref. 20. Assuming that the defect replaces an ion in the negatively charged sublattice, one can write this equation in the following approximate form

$$
\frac{4}{3 \pi} \frac{\left(\Omega_{0} a\right)^{3}}{v_{\perp}^{2} c} \frac{d}{\sqrt{\Omega_{l o c}^{2}-\Omega_{0}^{2}}}\left[\frac{\delta \beta}{\beta}-\left(\frac{m_{+}}{M}\right)^{2} \frac{\delta m}{\mu}\right]=1,
$$

where $\delta m$ is the deviation of the mass of the defect from that of the host atoms, $\delta \beta$ is the local change in the elastic constant, $M$ is the total mass of the positive $\left(m_{+}\right)$ and negative $\left(m_{-}\right)$ions, $\mu=m_{+} m_{-} /\left(m_{+}+m_{-}\right)$is their reduced mass, and $a$ denotes the lattice constant. This equation describes the LPM arising in the vicinity of the TO long-wavelength limiting frequency $\Omega_{0}$. Obviously, the real-value solution, $\Omega_{l o c}$, of Eq. (2) exists when the expression in the braces is positive.

The effect of LPM's on optical properties of crystals was studied in Refs. 22 24. The profile of electromagnetic-wave transmission is shown to have an asymmetric shape (Fano resonance) where the maximum is followed by a closely spaced zero. The maximum value of the transmission exponentially depends on the position of the defect in the crystal and, without absorption, it reaches unity for the defect placed at the center of the system. The width of the resonance decreases exponentially with an increase of the size of the system.

In spite of a general understanding that the local states should produce resonance tunneling, this result still seems surprising because transmission of light is affected by structural defects with microscopic dimensions much smaller than the wavelength of light. The physical explanation of this effect is based on the fact that the local polaritons emerge due to strong interaction between the electromagnetic field and local phonons. The latter have macroscopic dimensions comparable with the wavelength of IR light, thus making coupling with the external electromagnetic waves effective. As a result, the electromagnetic wave is carried through the sample by the phonons that tunnel resonantly due to the presence of the local state.

Because of the large spatial size of the local-polariton states, even at a very low impurity concentration, $\sim 10^{12}$ $\mathrm{cm}^{-3}$, they significantly overlap. As a result, an impurity polariton band (IPB) is formed inside the polariton band gap 25,26 This band has a number of interesting properties. For instance, the group velocity of electromagnetic excitations, propagating via such a band, has been found proportional to the concentration of the impurities, and it can be significantly smaller than the speed of light in vacuum. Also, for a large range of defect concentrations, the position of the boundaries of the IPB linearly depends 
on the frequency $\Omega_{l o c}$ of the "seed" LPM.26 Therefore, one can expect that the charge-state induced changes in the local elastic constants of the deep centers, that generate the IPB, will affect its boundaries in the same way as they affect the frequency of LPM at smaller concentrations. In the next section, we will explore this idea in reference to the well studied substitutional oxygen defect in gallium phosphide.

\section{CHARGE-STATE INDUCED CHANGES IN LOCAL ELASTIC CONSTANTS: $O_{P}$ CENTER IN GALLIUM PHOSPHIDE}

A striking alteration of the local elastic constants was established by Henry and Lang $\mathrm{H}$ in their detailed experimental studies of the charge states of the $O_{P}$ center in GaP. This deep donor center has two bound states, 1 and 2, with one or two bound electrons, correspondingly. Henry and Lang concluded that a significant decrease in the local lattice frequency after capture of the first or second electron is needed in order to consistently explain a variety of experimental data on photoionization and thermal emission (deep level transient spectroscopy) involving the two states in question. For state 2, where the second electron is trapped by or released from the electrically neutral center with a short-range attraction potential, this effect can be understood in the framework of the so called "zero-radius potential" model.6.27,28 (Such a model can be justified if the depth of the impurity potential well for the second electron at the center is small compared to its binding energy, $\epsilon_{T 2}$. U) It can be shown 2728 that for the "zero-radius potential" center the adiabatic potential curves $U(q)$, corresponding to bound and extended (continuum) electron states, would rather contact than intersect each other at the point $q_{\text {f }}$ (Fig. 2) where the electron binding energy goes to zero. (Here $q$ represents the configurational coordinate corresponding to a single mode of local vibrations that is coupled to the localized carrier(s).) Let us demonstrate that this rather general requirement accounts for the alteration of LVM of the $O_{P}$ center in $G a P$ introduced ad hoc in Ref. 6.

In the adiabatic harmonic single-mode approximation, the potential energy of the heavy ion, which itself is an eigenvalue of the light-electron Hamiltonian, can be presented as

$$
U_{N}(q)=\frac{\beta_{0} q^{2}}{2}+N \epsilon(q)+U_{c} n_{\uparrow} n_{\downarrow}+(2-N) E_{c} .
$$

The first term in this equation describes the elastic energy in the absence of the localized electrons with $\beta_{0}$ being the elastic constant, and

$$
\epsilon(q)=\epsilon_{0}-\lambda q-\frac{\gamma q^{2}}{2}
$$

is the localized-electron energy with electron-phonon coupling taken into account by expanding the electron energy in powers of $q$ about the equilibrium point in the absence of electrons. $U_{c}$ is the Hubbard repulsion energy for two electrons localized at the center, and the last term in Eq. (3) is the energy of the electron in the conduction band. $n_{\sigma}=0,1$ is the occupation number of a one-electron localized state with a spin $\sigma$, and $N=\sum_{\sigma} n_{\sigma}=0,1,2$ is the number of electrons trapped by the center. (In Eq. (4), we have chosen the negative sign at the quadratic term to assure the internal contact of terms $U_{1}(q)$ and $U_{2}(q)$; the sign of $\lambda$ is irrelevant.)

By using the contact condition at the point $q_{c}$,

$$
U_{1}\left(q_{c}\right)=U_{2}\left(q_{c}\right), \quad\left(\frac{d U_{1}}{d q}\right)_{q_{c}}=\left(\frac{d U_{2}}{d q}\right)_{q_{c}},
$$

it is easy to show that $E_{c}-\epsilon_{0}-U_{c}=\lambda^{2} / 2 \gamma$ and therefore the binding (thermal ionization) energy of state 2 is

$$
\epsilon_{T 2}=U_{1}\left(q_{1}\right)-U_{2}\left(q_{2}\right)=\frac{\lambda^{2}}{2 \gamma(1-x)(1-2 x)} .
$$

Here $q_{N}$ is the equilibrium configuration coordinate of the center with $N$ trapped electrons, and $x=\gamma / \beta_{0}$. By the same token, the optical ionization energy of state 2 is

$$
\epsilon_{\text {opt } 2}=U_{1}\left(q_{2}\right)-U_{2}\left(q_{2}\right)=\frac{\lambda^{2}}{2 \gamma(1-2 x)^{2}}
$$

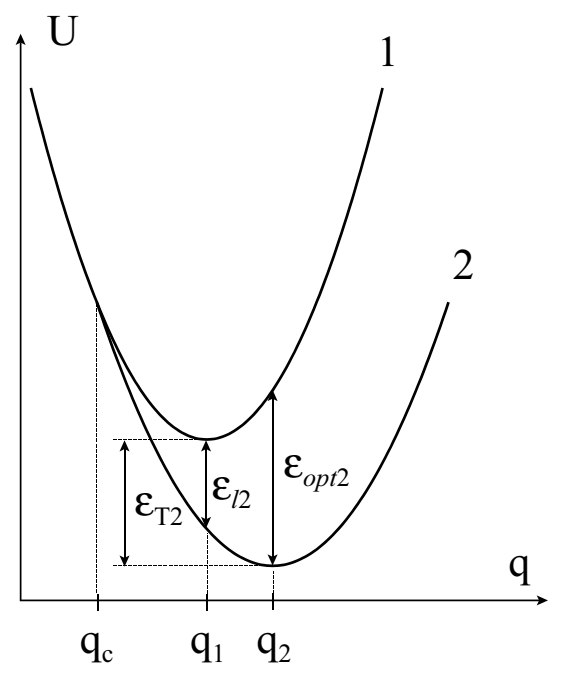

FIG. 2. Configuration coordinate diagram for the charge states $N=1,2$ of the $O_{P}$ deep center in $G a P$.

Then with experimentally measured values of $\epsilon_{T 2} \overline{\overline{7}}$ $0.89 \mathrm{eV}$ and $\epsilon_{\text {opt2 }}=2.03 \mathrm{eV}$ for the $O_{P}$ center in GaP,6 Eqs. (6) and (7) yield $x=\gamma / \beta_{0}=0.36$. This allows us to immediately evaluate the ratios of the LVM frequencies for different charge states of the $O_{P}$ center in GaP:

$$
\frac{\omega_{2}}{\omega_{1}}=\sqrt{\frac{1-2 x}{1-x}} \simeq 0.66 ; \quad \frac{\omega_{1}}{\omega_{0}}=\sqrt{1-x} \simeq 0.80
$$


in fairly good agreement with ratios $\omega_{2} / \omega_{1} \overline{\bar{n}} 0.65$ and $\omega_{1} / \omega_{0}=0.78$ extracted by Henry and Lang merous experimental data.

The goal of the above simple exercise is to show that if a multi-charge deep center in one of its states $(N=2)$ can be described by the "zero-radius potential" model, then non-linear electron-phonon coupling may result in a substantial change in the local elastic constants associated with this center when it captures or releases charge carriers. If the second electron is captured by the neutral center as a result of the radiative transition, the energy of the emitted photon is

$$
\epsilon_{l 2}=U_{1}\left(q_{1}\right)-U_{2}\left(q_{1}\right)=\frac{\lambda^{2}}{2 \gamma(1-x)^{2}} .
$$

From Eqs. (6), (7), and (9), the following criterion of applicability of the "zero-radius potential" model can be derived (see also Ref. ఫ):

$$
\epsilon_{T 2}=\sqrt{\epsilon_{o p t 2} \epsilon_{l 2}},
$$

as opposed to the standard relation 5

$$
\epsilon_{T 2}=\left(\epsilon_{o p t 2}+\epsilon_{l 2}\right) / 2 \text {, }
$$

held for deep centers with linear electron-phonon coupling when no change in the local frequencies is expected.

If the parameters of a deep center satisfyrelation (10), as it happens for the $O_{P}$ center in GaP the capture (release) of the first or second electron will diminish (increase) the local elastic constants in the vicinity of this center. To evaluate the effectiveness of such a rearrangement of the LVM's, let us consider a simple case of the $p$-type semiconductor doped by shallow acceptors with the concentration $N_{A}$ and partially compensated by the deep multi-charge donors of the type considered above with a concentration $N_{D} \ll N_{A}$. At equilibrium, all the deep donors will be free of electrons (state 0 ), i.e. positively charged. Then, incident light with photon energy close to $E_{g}-\epsilon_{o p t 1}$ will transfer electrons from the valence band to the states with $N=0$, thus recharging the deep centers $(+\rightarrow 0)$. (Here $E_{g}$ is the electron bandgap and $\epsilon_{\text {opt } 1}$ is the optical ionization energy of state 1.) Further evolution of the system depends onthe sign of the effective two-electron correlation energy 29,30

$$
U_{e f f}=U_{0}\left(q_{0}\right)+U_{2}\left(q_{2}\right)-2 U_{1}\left(q_{1}\right)=\epsilon_{T 1}-\epsilon_{T 2},
$$

where $\epsilon_{T 1}=U_{0}\left(q_{0}\right)-U_{1}\left(q_{1}\right)$ is the thermal ionization energy of state 1 .

By using experimental values 6 of $\epsilon_{T 1}=1.14 \mathrm{eV}$ and $\epsilon_{T 2}=0.89 \mathrm{eV}$, it is easy to find that $U_{\text {eff }} \simeq 0.26 \mathrm{eV}>0$ for the $O_{P}$ center in GaP. This means that, in this case, the electrically neutral state 1 generated by photons with energy $\left(E_{g}-\epsilon_{o p t 1}\right)$, which is close to $1.5 \mathrm{eV}$, 8 remains metastable under constant illumination conditions and will not be further converted into negatively charged state 2 . Then the electro-neutrality condition,

$$
p+N_{D}^{+}=N_{A}^{-},
$$

combined with the standard rate equations for the concentration $N_{D}^{0} \simeq N_{D}-N_{D}^{+}$of the deep neutral donors

$$
\partial N_{D}^{0} / \partial t=\sigma_{p 1}^{o p t} J N_{D}^{+}-p\left\langle v_{p}\right\rangle \sigma_{p 1}^{t h} N_{D}^{0}
$$

and for the concentration $N_{A}^{0}=N_{A}-N_{A}^{-}$of shallow neutral acceptors,

$$
\partial N_{A}^{0} / \partial t=p\left\langle v_{p}\right\rangle \sigma_{p A}^{t h} N_{A}^{-}-e_{p A} N_{A}^{0},
$$

allow one to evaluate the percentage of recharged deep centers. Here $p$ is the concentration of free holes, $\left\langle v_{p}\right\rangle$ is their mean thermal speed; $\sigma_{p 1}^{t h}\left(\sigma_{p A}^{t h}\right)$ is the nonradiative capture cross-section of the free holes by the deep neutral donors (shallow negative acceptors); $\sigma_{p 1}^{o p t}$ is the cross-section of the optical photo-neutralization of state $0 ; J$ is the flux of incident photons; $e_{p A}=$ $N_{v}\left\langle v_{p}\right\rangle \sigma_{p A}^{t h} \exp \left(-I_{A} / k_{B} T\right)$ is the rate of thermal emission of the holes by the shallow acceptors with the ionization energy $I_{A} ; N_{v}$ is the valence-band density of states; $k_{B}$ is the Boltzmann constant.

From Eq. (15) it follows that at not very low temperatures such that $T>I_{A}\left[k_{B} \ln \left(N_{v} / N_{A}\right)\right]^{-1}$, all the shallow acceptors are ionized, i.e. the concentration of the free holes [see Eq. (13)] $p$ is approximately $N_{A}\left(N_{A} \gg N_{D}\right)$. Then for the steady-state illumination conditions, Eq. (14) yields

$$
\frac{N_{D}^{0}}{N_{D}} \simeq\left[1+\frac{N_{A}\left\langle v_{p}\right\rangle \sigma_{p 1}^{t h}}{J \sigma_{p 1}^{o p t}}\right]^{-1}
$$

This means that the deep donors will be almost completely photo-neutralized $(+\rightarrow 0)$, if the flux of the incident sub-bandgap photons, $J \gtrsim 10^{19} \mathrm{~cm}^{-2} \mathrm{~s}^{-1}$. (For this estimate, we take $\sigma_{p 1}^{t h}=5 \times 10^{-21} \mathrm{~cm}^{-2}, \sigma_{p 1}^{\text {opt }} \overline{\bar{F}}$ $1.3 \times 10^{-16} \mathrm{~cm}^{-2}, N_{A}=10^{17} \mathrm{~cm}^{-3},\left\langle v_{p}\right\rangle=10^{7} \mathrm{~cm} / \mathrm{s}$. \&) Such a photon flux can be easily generated by a $1 W$ source for a spot area of the order of $1 \mathrm{~cm}^{2}$. In a $p$ type semiconductor with positive- $U_{\text {eff }}$ centers, this will convert a high-frequency LVM associated with these centers into a low-frequency one $\left(\omega_{0} \rightarrow \omega_{1}\right)$. However, for a $n$-type material the photo-neutralization of the deep positive- $U_{\text {eff }}$ centers will have the opposite effect: it converts the low-frequency LVM into the high-frequency one $\left(\omega_{2} \rightarrow \omega_{1}\right)$. If the concentration of the deep centers is high enough $\left(N_{D} \gg N_{A}\right)$, then the light from the sub-bandgap or fundamental regions will convert the intermediate-frequency LVM into the high and lowfrequency LVM's: $\omega_{1} \rightarrow \omega_{0}, \omega_{1} \rightarrow \omega_{2}$ [see Fig. 3a and Eq. (8)].

For materials with negative- $U_{\text {eff }}$ centers, the continuous sub-bandgap (impurity) illumination should, in principle, have more a profound fffect on LVM due to the so called disproportionation 9 i.e. thermodynamically driven spontaneous decay of the metastable electron state 
1 into the states with the next higher, state 2 , and the next lower, state 0 , number of electrons. In $p$-type materials, it will initially convert the high-frequency LVM, $\omega_{0}$, into the low-frequency one, $\omega_{1}$, associated with the neutral donors, which later on due to $U_{\text {eff }}<0$ will be further spontaneously converted into the lower one, $\omega_{2}$. For $n$ type materials, as in the previous case of positive $U_{\text {eff }}$, the effect will be opposite: $\omega_{2} \rightarrow \omega_{1} \rightarrow \omega_{0}$ (Fig. $3 \mathrm{~b}$ ). And, finally, in semi-insulating material, whep the Fermi level is pinned by the negative- $U_{\text {eff }}$ defects, 29.30 the illumination should convert the lowest and highest energy LVM into the intermediate one: $\omega_{2} \rightarrow \omega_{1}, \omega_{0} \rightarrow \omega_{1}$ (Fig. $3 \mathrm{~b})$.

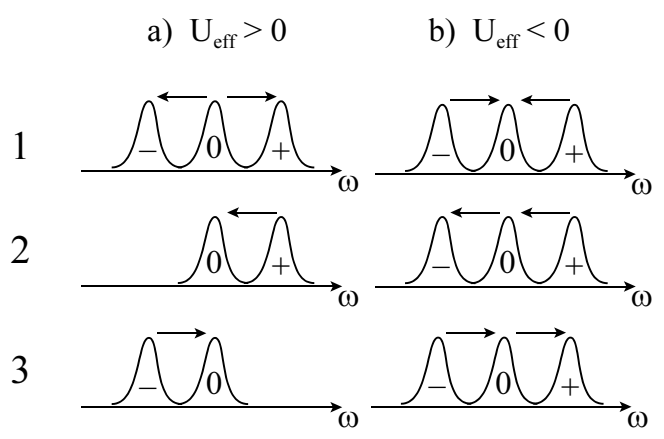

FIG. 3. Sub-bandgap light-absorption-induced modification of LVM's for the different charge states of a deep center with (a) positive and (b) negative effective two-electron correlation energy in the semi-insulating (1), p-type (2), and n-type (3) semiconductors.

\section{CENTERS AND MATERIALS PERSPECTIVE FOR ALTERATION OF LPM'S}

It is interesting to compare predictions based on our simple model with the observed optically induced conver-

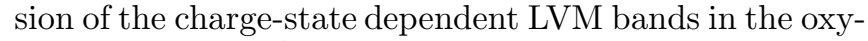
gen doped GaAs 9 It has been proven that the off-center substitutional $O_{A s}$ in GaAs represents one of the few negative- $U_{\text {eff }}$ systems in compound semiconductors 9 , 20 And indeed, during illumination with sub-bandgap light with photon energy around $1.37 \mathrm{eV}$, the high-energy $730.7 \mathrm{~cm}^{-1}$ band $A$ is almost completely converted into the low-energy $714.9 \mathrm{~cm}^{-1}$ band $B$ through the other low-energy $714.2 \mathrm{~cm}^{-1}$ band $B^{\prime}$. These bands were attributed, correspondingly, to the unoccupied, singly, and doubly occupied electron states of the $O_{A s}$ center in certain semi-insulating samples of $G a A s$, where the Fermi level was pinned below the lowest, doubly occupied, state 2 of $O_{A s} .10$ The material in question is analogous to $p$-type semiconductors with the negative- $U_{\text {eff }}$ centers in our classification. We conclude that, though in accordance with our model $\omega_{0}>\omega_{2}$, the frequency of the singly occupied state, $\omega_{1}$, is almost the same as that of the doubly occupied one, $\omega_{2}$, as opposed to the case of the $O_{P}$ center in $G a P$ for which $\omega_{0}^{2}-\omega_{1}^{2}=\omega_{1}^{2}-\omega_{2}^{2}>0$. An extensive discussion of the electronic structure of $O_{A s}$ in $\mathrm{GaAs}$ can be found in Ref. 10. According to Skowronski,10 oxygen in GaAs creates a complex offcenter defect with dangling gallium hybrids involved, whose coupling strongly depends on the charge state of the center. The adiabatic potentials of the coupled dangling bonds in tetrahedrally bonded and amorphous semiconductors are shown to have a complex multi-well structure that is extremely sensitive to parameters of the structural defects in question.31 33 For instance, a stretched bond of the type, participating in the formation of $O_{A s}$ in $G a A s$, with one or three electrons, will be strongly coupled to at leasf two LVM's with chargestate dependent frequencies.32 Though such a defect is not described by the above single-mode model, it can be responsible for photo-induced changes in the local elastic constants.

Let us now return to $O_{P}$ in $\mathrm{GaP}$. The elastic constants for the three different charge states, $\beta_{N}(N=0,1,2 \mathrm{enu}-$ merates the charge states of the center), of this center are directly related to the frequency of the correspondent LVM's given by Eq. (8):

$$
\frac{\beta_{2}}{\beta_{1}}=\left(\frac{\omega_{2}}{\omega_{1}}\right)^{2} ; \quad \frac{\beta_{1}}{\beta_{0}}=\left(\frac{\omega_{1}}{\omega_{0}}\right)^{2} .
$$

We want to check if this defect can give rise to LPM's in any of its charge states. According to Eq. (2), the criterion for the appearance of LPM's is set by

$$
\frac{\delta \beta_{N}}{\beta}-\left(\frac{m_{+}}{M}\right)^{2} \frac{\delta m}{\mu}>0,
$$

where $\delta \beta_{N}=\beta_{N}-\beta$ with $\beta$ being the elastic constant of the host atom. Therefore, it is not sufficient just to know the ratios of the local elastic constants, we need to establish their values. It is equivalent to finding the frequencies of LVM's since $\beta_{N} \simeq m_{O} \omega_{N}^{2}$, where $m_{O}$ is the mass of the oxygen atom. Direct experimental measurements of these frequencies are not available to us; however, it is possible to determine them from the configuration coordinate diagram of this center (Fig. 2). Calculated by means of the "zero-radius potential" model, the multi-phonon emission electron-capture crosssections were compared in Ref. 7 with the experimental data from deep level transient spectroscopy.6 It gave $\omega_{1} \simeq 195 \mathrm{~cm}^{-1}$ for the $O_{P}$ defect in $G a P$. This value differs from the one $\left(\omega_{1} \simeq 155 \mathrm{~cm}^{-1}\right)$ obtained in the original paper (Ref. 6) where fitting is believed to be somewhat inconsistent. Later, in Ref. 34 it was argued that because of a strong field dependence of the thermal emission energy, $\epsilon_{T 1}$, of the electron from the singly occupied state, the deep level transient spectroscopy data should be re-evaluated. This led to a modified value of the local-phonon energy $\omega_{1} \simeq 290 \mathrm{~cm}^{-1}$. We believe that the above re-evaluation of $\omega_{1}$ should not affect the ratios 
of the frequencies given by Eq. (8), for in the framework of the model developed, it is based on the value of parameter $x=\gamma / \beta_{0}=0.36$. The latter, in turn, was obtained by means of the ratio $\epsilon_{o p t 2} / \epsilon_{T 2}$ involving experimentally measured ionization energies of the state $N=2$ with a short-range potential that can hardly be affected by the electric field in the area of the p-n junction.

Examining criterion (17) for LPM's to occur, one can see that in the most favorable case $\delta \beta_{N}$ should be positive while $\delta m$ should be negative. This has a simple physical explanation. As the frequency of the TO mode is defined by $\omega_{T O}=(\beta / \mu)^{1 / 2}$, in order to make the defect frequency $\Omega_{\text {loc }}^{(N)}$ advance into the polariton bandgap (above the TO frequency) one should either make $\beta_{N}$ larger than $\beta(\simeq 175 \mathrm{~N} / \mathrm{m}$ in gallium phosphide) of the host atoms, or decrease the mass of the defect.

For the case of the oxygen center in gallium phosphide where $\delta m<0$, we examined three values of $\beta_{1}$ obtained by means of all three values of $\omega_{1}$ from Refs. 6,7,34. Then we used ratios (8) between the rest of the LVM frequencies to determine the local elastic constants $\beta_{N}$ of the defect in three charge states. We find that using the data from Refs. 6.7, it is not possible to satisfy our condition Eq. (17). On the other hand, $\omega_{1} \simeq 290 \mathrm{~cm}^{-1}$, obtained in Ref. 34, results in $\beta_{0} \simeq 160 \mathrm{~N} / \mathrm{m}, \beta_{1} \simeq 95 \mathrm{~N} / \mathrm{m}$, and $\beta_{2} \simeq 40 \mathrm{~N} / \mathrm{m}$. Even though for all three charge states $\delta \beta_{N}<0, \beta_{N=0}$ still satisfies Eq. (17). Thus, we predict that in the p-type (semi-insulating) GaP:O, the LPM associated with $N=0$ charge-state will be eliminated (created) by illuminating the sample with the light with a photon energy close to $1.48 \mathrm{eV}(0.96 \mathrm{eV})$ (see Ref. 6 for details), and as it was shown for the charge-state dependent LVM's, this process is reversible. We also stress that the LPM's arise/disappear between TO $\left(365 \mathrm{~cm}^{-1}\right)$ and LO $\left(405 \mathrm{~cm}^{-1}\right)$ frequencies, in contrast to the LVM's that occur either below or above this region.

The oxygen defect in $G a P$ cannot, by any means, be considered as a single candidate for tunable LPM's to be observed. Semiconductors and insulators that possess a complete (omnidirectional) polariton gap include well known materials such as $\mathrm{GaP}, \mathrm{SiC}, \mathrm{ZnS}, \mathrm{ZnTe}, \mathrm{CuI}, \mathrm{CaF}_{2}, \mathrm{SrF}_{2}, \mathrm{BaF}_{2}, \mathrm{PbF}_{2}, 35$ as well as extensively studied nitrides $A l N, G a N, \operatorname{InN}$. 37 In these materials many impurities form deep centers. 3840 The charge-state dependent LVM's, which can be considered as precursors of tunable LPM's, remain relatively unstudied. In a recent paper by Wetzel et al, , $^{3}$ the charge-state dependent triplet of LVM's generated by oxygen in $G a N$ has been reported, which is similar to the GaP:O deep center. Even though this defect does not satisfy our condition for LPM's, Eq. (17), $(\delta \beta<0$ and $\delta m>0$ for this defect), it gives us confidence that some defects can give rise to LPM's in crystals with the complete polariton bandgap. Our optimism is also supported by the fact that there are many defects that satisfy this criterion but occurin materials without a polariton gap namely: GaAs:O, 10 EL2 center in GaAs, 14 GaAs:Si, 10
$S i: H, 17$ and $A l A s: B e, 18$ Si:C 11,12

To summarize, we have shown that a possibility exists to effectively control the optical properties, in particular, light transmission, of polar crystals in the far IR region in the vicinity of the polariton bandgap, by modifying the charge state of the deep center by means of light from the visible or near IR region. To obtain more specific results, further experimental and theoretical studies are needed.

\section{ACKNOWLEDGMENTS}

We are indebted to S. Schwarz for reading and commenting on the manuscript. This work was partially supported by the NATO Linkage Grant N974573, CUNY Collaborative Grant, and PSC-CUNY Research Award, as well as by the NSF grant DMR-0071823 and the Nelson grant (SDSMT).

${ }^{1}$ S. T. Pantelides, in Deep Centers in Semiconductors, ed. by S. T. Pantelides, 2nd edition (Gordon and Breach, New York, 1992), p.1.

${ }^{2}$ G. F. J. Garlick, Reports on Progress in Physics, London, 1967, v. 30, part 2, p. 491.

${ }^{3}$ P. T. Landsberg, Physica Status Solidi 41, 457 (1970).

${ }^{4}$ M. Lax, Phys. Rev. 119, 1502 (1960).

${ }^{5}$ K. Huang and A. Rhys, Proc. Royal Soc. A 204, 406 (1950).

${ }^{6}$ C. H. Henry and D. V. Lang, Phys. Rev. B 15, 989 (1977).

${ }^{7}$ V. N. Abakumov, I. A. Merkulov, V. I. Perel', and I. N. Yassievich, Zh. Eksp. Teor. Fiz. 89, 1472 (1985) [Sov. Phys. JETP 62, 853 (1985)].

${ }^{8}$ P. J. Dean, in Deep Centers in Semiconductors, ed. by S. T. Pantelides, 2nd edition (Gordon and Breach, New York, 1992), p.215.

${ }^{9}$ H. Ch. Alt, Phys. Rev. Lett. 65, 3421 (1990).

${ }^{10}$ M. Skowronski, in Deep Centers in Semiconductors, ed. by S. T. Pantelides, 2nd edition (Gordon and Breach, New York, 1992), p.379.

${ }^{11}$ E. V. Lavrov, B. B. Nielsen, J. R. Byberg, B. Hourahine, R. Jones, S. Öberg, and P. R. Briddon, Phys. Rev. B 62, 158 (2000).

${ }^{12}$ E. V. Lavrov, L. Hoffmann, and B. B. Nielsen, Phys. Rev. B 60, 8081 (1999).

${ }^{13}$ C. Wetzel, H. Amano, I. Akasaki, J. W. Ager III, I. Gregory, M. Topf, and B. K. Meyer, Phys. Rev. B 61, 8202 (2000).

${ }^{14}$ W. K. Ge, C. Y. Song, and D. S. Jiang, Phys. Rev. B 53, 9809 (1996).

15 Zh.-G. Wang, L.-A. Ledebo, and H. G. Grimmeiss, J. Phys. C 17, 259 (1984).

${ }^{16}$ J. A. Wolk, M. B. Kruger, J. N. Heyman, W. Walukiewicz, R. Jeanloz, and E. E. Haller, Phys. Rev. Lett. 66, 774 (1991). 
17 V. P. Markevich, M. Suezawa, and L. I. Murin, J. Appl. Phys. 84, 1246 (1996).

${ }^{18}$ H. Ono, and T. Baba, Phys. Rev. B 47, 16628 (1993).

${ }^{19}$ L. I. Deych and A. A. Lisyansky, Bull. Amer. Phys. Soc. 42, 203 (1997); Phys. Lett. A, 240, 329 (1998).

${ }^{20}$ V. S. Podolsky, L. I. Deych, and A. A. Lisyansky, Phys. Rev. B, 57, 5168 (1998).

${ }^{21}$ V. I. Rupasov and M. Singh, Phys. Rev. A, 54, 3614 (1996); Phys. Rev. A 56, 898 (1997).

${ }^{22}$ L. I. Deych and A. A. Lisyansky, Phys. Lett. A, 243, 156 (1998).

${ }^{23}$ L. I. Deych, A. Yamilov and A. A. Lisyansky, Europhys. Lett., 46, 534 (1999).

${ }^{24}$ L. I. Deych, A. Yamilov and A. A. Lisyansky, Phys. Rev. B, 59, 11339 (1999).

${ }^{25}$ A. Yamilov, L. I. Deych, and A. A. Lisyansky, J. Opt. Soc. Amer. B 17, 1498 (2000).

${ }^{26}$ L. I. Deych, A. Yamilov, and A. A. Lisyansky, Phys. Rev. B, Phys. Rev. B 62, 6301 (2000).

27 A. I. Baz', Ya. B. Zeldovich, and A. M. Perelomov, Scattering, Reactions and Decay in Nonrelativistic Quantum Mechanics, IPST, Jerusalem, 1969.

${ }^{28}$ S. V. Meshkov, Zh. Eksp. Teor. Fiz. 89, 1734 (1985) [Sov. Phys. JETP 62, 1000 (1985)]

${ }^{29}$ P. W. Anderson, Phys. Rev. Lett. 34, 953 (1975).
${ }^{30}$ N. F. Mott and E. A. Davis, Electronic Processes in NonCrystalline Materials, 2nd ed., Clarendon Press, Oxford (1979).

31 A. G. Petukhov, A. V. Radchik, and M. G. Foygel, Pis'ma Zh. Eksp. Teor. Fiz. 41, 502 (1985) [JETP Lett. 41, 612 (1985)].

32 A. G. Petukhov and M. G. Foygel, Fiz. Tekh. Poluprovodn. 21, 118 (1987) [Sov. Phys. Semicond. 21, 71 (1987)].

${ }^{33}$ M. Foygel, A. G. Petukhov, and A. S. Andreyev, Phys. Rev. B 48, 17018 (1993).

${ }^{34}$ U. S. Qurashi, M. Z. Iqbal, C. Delerue, and M. Lannoo, Phys. Rev. B 23, 13331 (1992).

${ }^{35}$ H. Bilz, W. Kress, Phonon Dispersion Relations in Insulators, Springer-Verlag, New York, 1979.

${ }^{36}$ Semiconductors. Group IV elements and III-V Compounds, ed. by O. Madelung, Springer-Verlag, New York, 1991.

${ }^{37}$ F. Bechstedt, U. Grossner, and J. Furthmuller, Phys. Rev. B 62, 8003 (2000).

38 A. S. Barker, Jr. and A. J. Sievers, Rev. Mod. Phys. 47, Suppl. 2 (1975).

${ }^{39}$ Imperfections in III/V materials, ed. by E. R. Weber, Semiconductor and Semimetal series, vol. 38, Academic Press, San Diego, 1993.

${ }^{40}$ R. C. Newman Infra-red Studies of Crystal Defects, Taylor and Francis, London, 1973. 